\title{
Estimations des doses dans le cadre d'une étude comparative des voies radiales et fémorales droites dans la technique radiologique coronarographique
}

\author{
T. BLANPAIN ${ }^{1,2}$, C. BRASSELET ${ }^{1}$, S. TASSAN-MANGINA ${ }^{1}$, \\ A. DESCHILDRE ${ }^{1}$, J.P. CLÉMENT ${ }^{2}$, N. GAILLOT-PETIT ${ }^{2}$ \\ C. TOURNEUX ${ }^{2}$, D. METZ ${ }^{1}$
}

(Manuscrit reçu le 19 novembre 2007, accepté le 5 avril 2008)

RÉSUMÉ L'irradiation des patients et de l'opérateur au cours de coronarographie et/ou angioplastie coronaire, fait désormais partie des préoccupations légitimes de la cardiologie interventionnelle moderne. Les voies fémorales et radiales droites sont les voies d'accès privilégiées pour la réalisation de ces examens. Néanmoins, peu de registres comparent ces 2 voies d'abord en terme d'irradiation du patient et de l'opérateur. L'objectif de ce registre est une évaluation comparative de ces 2 techniques. 423 patients consécutifs ayant bénéficié dans notre centre de l'une ou l'autre de ces techniques réalisées entre octobre 2005 et mars 2006 ont été inclus. Les paramètres retenus sont les suivants : dose bras gauche opérateur, produit dose $\times$ surface pour le patient, temps global des procédures et durée de la scopie. Pour les 4 paramètres étudiés, les résultats sont significativement supérieurs en cas de coronarographie et coronarographie plus angioplastie ad hoc par voie radiale que par voie fémorale. Respectivement : (1) temps de scopie : 4 min versus $1,8 \mathrm{~min}$, et 9,6 min versus $6,3 \mathrm{~min}$; (2) temps de procédure : 15 min versus 9,5 , et 41 min versus $35,25 \mathrm{~min}$; (3) dose patient : $59 \mathrm{~Gy} \mathrm{~cm}^{2}$ versus $37,5 \mathrm{~Gy} \mathrm{~cm}^{2}$, et $123 \mathrm{~Gy} \mathrm{~cm}^{2}$ versus $102 \mathrm{~Gy} \mathrm{~cm}^{2}$; (4) dose opérateur: $29 \mu \mathrm{Sv}$ versus $13 \mu \mathrm{Sv}$, et $69 \mu \mathrm{Sv}$ versus $40 \mu \mathrm{Sv}$. Malgré son intérêt en terme d'économie de complication de voie d'abord, la voie radiale droite est significativement plus irradiante pour les patients et les opérateurs que la voie fémorale droite.

ABSTRACT Doses estimations of a comparative study between radial and femoral access during coronary angiograms and percutaneous coronary interventions.

Irradiation of operators and patients during coronary angiograms (CA) and percutaneous coronary interventions (PCI) are legitimate matter of concern of modern interventional cardiology. Femoral and right radial approaches are privileged accesses for the CA and PCI. However, few registers compare these 2 accesses in term of operators and patients' irradiation. The purpose of this register is to establish a comparative evaluation between these 2 techniques. 423 consecutive patients having benefited in our center either one or the other of these techniques performed between October, 2005 and March, 2006 were included. The retained parameters are: Left operator's arm dose, patient's surface dose, global procedure time and scopy time. The results are significantly higher in radial CA and PCI ad hoc

Laboratoire de cathétérisme cardiaque, département de cardiologie, Hôpital Robert Debré, 51100 Reims, France.

Unité de Radio physique médicale et de Radioprotection, Hôpital Robert Debré, 51100 Reims, France. 


\begin{abstract}
than in femoral access for the four studied parameters: (1) scopy time: 4 min versus $1.8 \mathrm{~min}$, and $9.6 \mathrm{~min}$ versus $6.3 \mathrm{~min}$; (2) procedure time: 15 min versus 9.5 , and

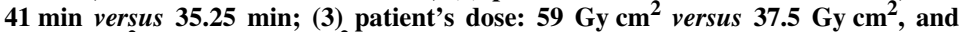
$123 \mathrm{~Gy} \mathrm{~cm}^{2}$ versus $102 \mathrm{~Gy} \mathrm{~cm}^{2}$; (4) operator's dose: $29 \mu \mathrm{Sv}$ versus $13 \mu \mathrm{Sv}$, and $69 \mu \mathrm{Sv}$ versus $40 \mu \mathrm{Sv}$. In spite of its interest with less post-operative vascular complications, the right radial access is significantly more irradiating for the operators and the patients than the femoral access.
\end{abstract}

Keywords: Percutaneous coronary intervention / radial and femoral artery / patient and operator radiation exposure

\title{
1. Introduction
}

Longtemps ignorée par les cardiologues interventionnels, la problématique de l'irradiation des patients et de l'opérateur en cours de coronarographies et/ou d'angioplasties coronaires, et son corollaire immédiat la radioprotection, fait désormais partie des préoccupations légitimes de la cardiologie interventionnelle moderne (Von Boetticher et al., 2003).

Les voies fémorales et radiales droites sont les voies d'accès privilégiées pour la réalisation de coronarographies et/ou d'angioplasties coronaires. Néanmoins, peu de registres comparent ces deux méthodes en terme d'irradiation du patient et de l'opérateur. Nous avons donc réalisé un registre monocentrique, non randomisé mais effectué en aveugle pour l'opérateur, afin de faire une évaluation comparative de ces deux techniques.

\section{Matériel et méthodes}

\subsection{Conception de l'étude}

Quatre cent vingt et un patients consécutifs ayant bénéficié dans notre centre d'une coronarographie et/ou d'une angioplastie coronaire réalisée entre octobre 2005 et mars 2006 ont été inclus comme suit :

- dans le groupe «coronarographie seule», 149 voies radiales versus 98 fémorales ;

- dans le groupe « coronarographie plus angioplastie $a d$ hoc », 90 radiales versus 84 fémorales.

Le choix de la voie d'abord s'est fait en aveugle pour l'opérateur mais toujours avec le souci d'avoir une manœuvre d'Allen positive pour la voie radiale (la manœuvre d'Allen est une manœuvre qui permet de vérifier si l'arcade artérielle palmaire est perméable afin de vasculariser la main entièrement par l'artère cubitale si il advenait que l'artère radiale utilisée pour le cathétérisme soit occluse à son issue, ce qui survient dans $5 \%$ des cas). 
À noter que quatre opérateurs ont participé à ce registre et que chacun d'eux possède une expérience de plus de cinq ans de coronarographie et/ou d'angioplastie coronaire par voie fémorale et/ou radiale.

Les critères de non inclusion étaient (1) un syndrome coronaire aigu avec sus décalage du segment ST, l'existence préalable de pontages aorto-coronariens, un cathétérisme droit ou toute autre exploration vasculaire associée qui risquaient de provoquer un biais au comparatif de par leur divergence de procédure, et (2) une intervention réalisée par 2 opérateurs (effectivement la formation d'opérateur ne devait pas entrer en ligne de compte pour ne pas créer de biais à l'étude).

Ont été inclus des procédures de coronarographies seules (ventriculographie et angiographie coronaire) et des coronarographies + angioplastie ad hoc (angioplastie d'un vaisseau dans la foulée), toutes autres procédures ont été exclues.

Par conséquent seulement 421 patients sur 847 patients au total ont été inclus.

\subsection{L'environnement radiologique}

L'appareillage radiologique de notre service est composé de deux salles identiques (âgées respectivement de 9 et 10 ans) de type Integris HM 3000, version 2 (Philips, Netherland) équipée chacune d'un tube SUPER ROTALIX céramique MRC et d'un générateur RX DE $100 \mathrm{~kW}$. L'amplificateur de brillance est doté de trois champs $(13,18$ ou $23 \mathrm{~cm}$ ) ; la résolution matricielle est de $512 \times 512$ (ventricule gauche) et de $1024 \times 1024$ (coronaires); les cadences utilisées sont de 25 images/s pour le ventricule gauche et de 12,5 images/s pour les coronaires. La scopie est de type pulsée (Photos 1 et 2).

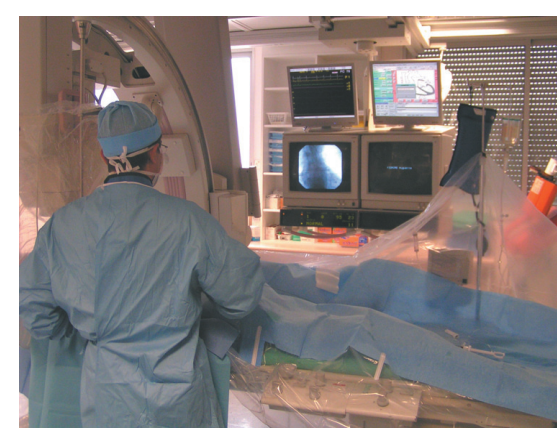

Photo 1.

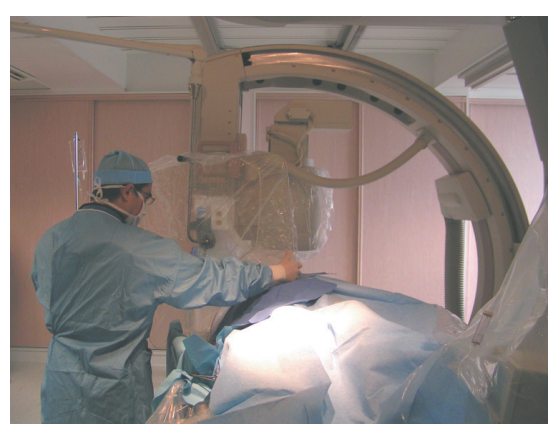

Photo 2. 


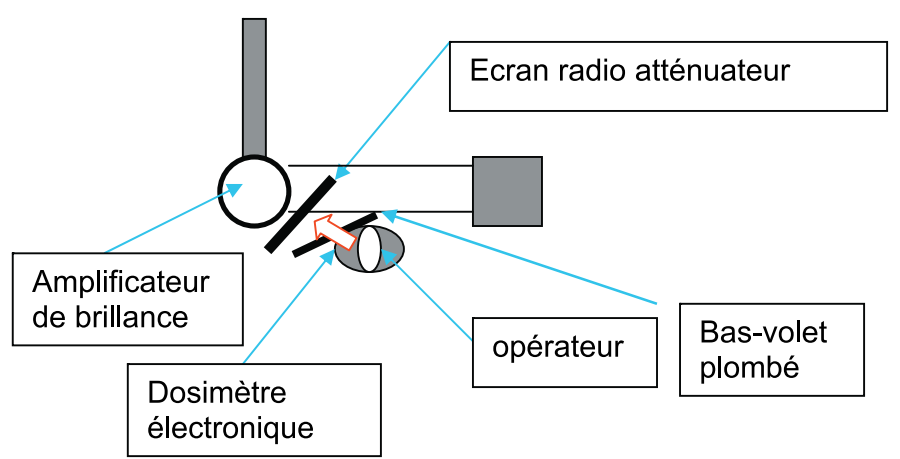

Figure 1 - Positionnement de l'opérateur et angulation du dosimètre.

Operator's position and angulation of dosimeter.

\subsection{Les conditions de mesures et contexte dosimétrique}

Pour l'opérateur, les mesures de la dose ont été réalisées à l'aide de dosimètres électroniques à détecteur type diode silicium fixés sur son bras gauche (au plus proche de l'amplificateur de brillance, et de façon à inhiber le phénomène de réponse angulaire du dosimètre électronique) (Photo 3 et Fig. 1).

On notera la sensibilité des dispositifs de mesure: pour le dosimètre électronique à diode silicium $1 \mu \mathrm{Sv}$ et pour le film dosimètre de $0,1 \mathrm{mSv}$

Le seuil de réponse en énergie du dosimètre électronique est de $15 \mathrm{keV}$ (largement dans les gammes d'énergie de la radiologie).

Par ailleurs le suivi réglementaire de l'opérateur était assuré par la dosimétrie opérationnelle (dosimètre électronique) et la dosimétrie passive (film dosimètre) poitrine habituelle sous la chasuble plombée (Photo 4).

Pour le patient, les mesures ont été réalisées par l'intermédiaire d'une chambre à ionisation (type DIAMENTOR) présente sur l'appareil de radiologie numérique (Integris HM 3000). Cette chambre permet de mesurer le produit dose $\times$ surface (PDS). Remarquons qu'au moment de l'étude, l'étalonnage régulier de ces chambres n'était pas encore obligatoire.

\subsection{Le contexte de radioprotection}

La protection de l'opérateur était assurée par le port d'une chasuble plombée équivalent $0,5 \mathrm{~mm}$ de plomb en ventral et $0,25 \mathrm{~mm}$ de plomb en dorsal, d'un cache 


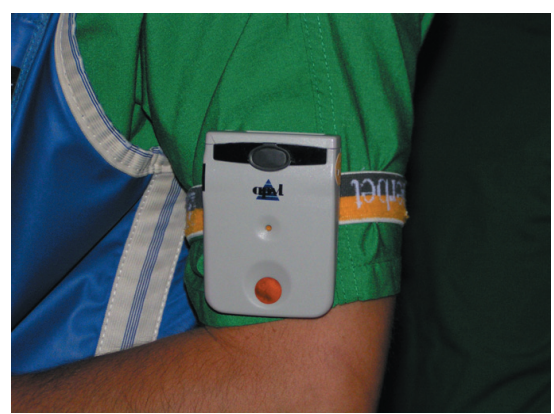

Photo 3.

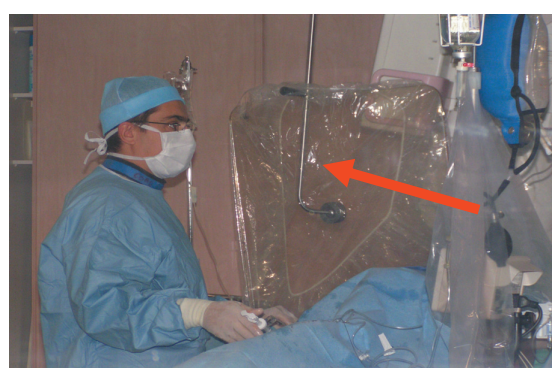

Photo 5.

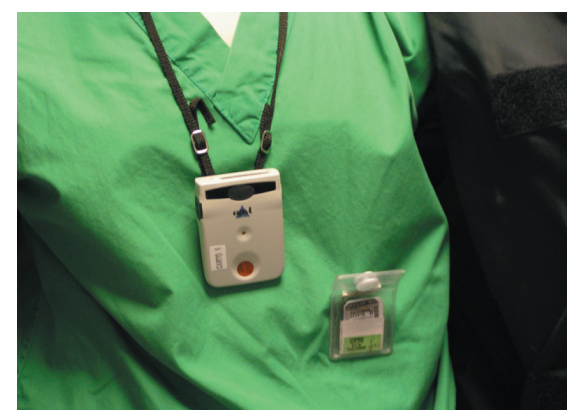

Photo 4.

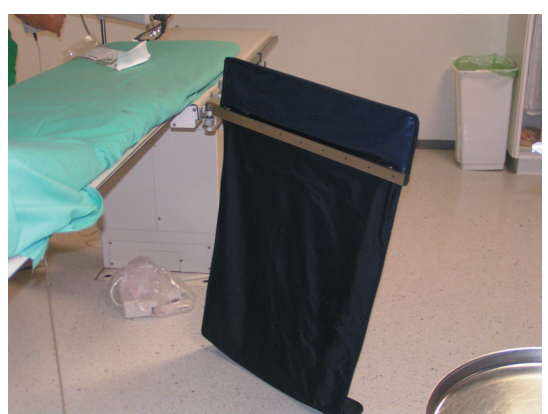

Photo 6.

thyroïde et par l'utilisation d'un bas volet plombé et d'une vitre plombée équivalents $0,5 \mathrm{~mm}$ de plomb ( $c f$. Photos 5 et 6 ).

\subsection{Recueil de données}

À chaque procédure plusieurs paramètres ont été relevés: l'exposition de l'opérateur avec le dosimètre électronique du bras ( $\mu \mathrm{Sv}$ ) (Photo 3 ) et le dosimètre poitrine $(\mu \mathrm{Sv})$ (Photo 4$)$, le produit dose $\times$ surface caractérisant l'exposition délivrée au patient ou PDS $\left(\mathrm{Gy} \mathrm{cm}^{2}\right.$ ) (Photo 7$)$, les temps de procédures et de scopie ( $\mathrm{min})$ ainsi que l'index de masse corporelle des patients ou BMI $\left(\mathrm{kg} / \mathrm{cm}^{2}\right)$.

\section{Résultats}

Les résultats sont analysés sur deux groupes d'examens :

- le groupe «coronarographie seule »: procédure strictement diagnostique (247 patients);

- le groupe «coronarographie + angioplastie ad hoc»: phase diagnostique suivie d'un geste thérapeutique (174 patients). 


\section{TABLEAU I}

Groupe coronarographie seule. Valeurs exprimées comme médianes. Valeurs [mini-max]. Coronary angiogram alone. Values expressed as med [mini-max] values.

\begin{tabular}{|c|c|c|c|c|}
\hline$\frac{\text { Coro seule }}{\text { Médiane }}$ & $\begin{array}{c}\text { Fémoral } \\
(98 \text { patients })\end{array}$ & $\begin{array}{c}\text { Radial } \\
(149 \text { patients })\end{array}$ & $\mathbf{p}$ & $\begin{array}{l}\text { \% d'augmentation de la voie radiale } \\
\text { par rapport à la voie fémorale }\end{array}$ \\
\hline $\begin{array}{l}\text { Dose patient } \\
\left(\mathrm{Gy} \mathrm{cm}^{2}\right)\end{array}$ & $\begin{array}{c}37,5 \\
{[10-130]}\end{array}$ & $\begin{array}{c}59 \\
{[15-334]}\end{array}$ & $<0,0001$ & 36,44 \\
\hline $\begin{array}{l}\text { Dose bras opérateur } \\
(\mu \mathrm{Sv})\end{array}$ & $\begin{array}{c}13 \\
{[1-164]}\end{array}$ & $\begin{array}{c}29 \\
{[1-195]}\end{array}$ & $=0,0001$ & 55,17 \\
\hline $\begin{array}{l}\text { Temps de scopie } \\
(\mathrm{min})\end{array}$ & $\begin{array}{c}1,8 \\
{[0,5-15,5]}\end{array}$ & $\begin{array}{c}4 \\
{[1-28]}\end{array}$ & $<0,0001$ & 55 \\
\hline $\begin{array}{l}\text { Temps de procédure } \\
\text { (min) }\end{array}$ & $\begin{array}{c}9,5 \\
{[2-35]}\end{array}$ & $\begin{array}{c}15 \\
{[4-60]}\end{array}$ & $<0,0001$ & 36,67 \\
\hline
\end{tabular}

$\mathrm{Nb}: \mathrm{p}<0,05$ pour être significatif.

TABLEAU II

Groupe coronarographie + angioplastie ad hoc. Valeurs exprimées comme médianes. Valeurs [mini-max].

Coronary angiogram followed by ad-hoc PCI. Values expressed as med [mini-max] values.

\begin{tabular}{|c|c|c|c|c|}
\hline $\begin{array}{l}\text { Coro + angioplastie ad hoc } \\
\text { Médiane } \\
\text { Valeur [min,max] }\end{array}$ & $\begin{array}{c}\text { Fémoral } \\
\text { (84 patients) }\end{array}$ & $\begin{array}{c}\text { Radial } \\
\text { (90 patients) }\end{array}$ & $\mathbf{p}$ & $\begin{array}{l}\text { \% d'augmentation de la voie } \\
\text { radiale par rapport à la voie } \\
\text { fémorale }\end{array}$ \\
\hline $\begin{array}{l}\text { Dose patient } \\
\left(\mathrm{Gy} \mathrm{cm}^{2}\right)\end{array}$ & $\begin{array}{c}102 \\
{[23-383]}\end{array}$ & $\begin{array}{c}123 \\
{[21-398]}\end{array}$ & $=0,006$ & 17,07 \\
\hline Dose bras opérateur ( $\mu \mathrm{Sv})$ & $\begin{array}{c}40 \\
{[2-275]}\end{array}$ & $\begin{array}{c}69 \\
{[4-531]}\end{array}$ & $=0,043$ & 42,03 \\
\hline Temps de scopie (min) & $\begin{array}{c}6,3 \\
{[1,4-30,9]}\end{array}$ & $\begin{array}{c}9.6 \\
{[2,9-34,8]}\end{array}$ & $<0,0001$ & 34,38 \\
\hline Temps de procédure (min) & $\begin{array}{c}35.25 \\
{[12,5-103]}\end{array}$ & $\begin{array}{c}41 \\
{[13-95]}\end{array}$ & $=0,048$ & 14,02 \\
\hline
\end{tabular}

$\mathrm{Nb}: \mathrm{p}<0,05$ pour être significatif.

Le tableau I compare la voie radiale droite à la voie fémorale droite en ce qui concerne le PDS, la dose bras opérateur, le temps de scopie et le temps de procédure respectivement pour le groupe coronarographie seule, et pour le groupe coronarographie + angioplastie ad hoc.

$\mathrm{Nb}$ : les valeurs sont exprimées en valeurs médianes suivies de l'écart type.

Le temps de scopie est significativement plus long lors d'une coronarographie et d'une coronarographie puis angioplastie ad hoc par voie radiale que par voie fémorale $(1,8 \pm 4,7$ min versus $2 \pm 2,0 \mathrm{~min} ; \mathrm{p}<0,0001$ et $6,3 \pm 6,2 \mathrm{~min}$ versus $9,6 \pm 4,8 \mathrm{~min} ; \mathrm{p}<0,0001$, respectivement). 
Le temps de procédure est aussi significativement plus long en cas de coronarographie et coronarographie plus angioplastie ad hoc par voie radiale que par voie fémorale $(15 \pm 9,6$ min versus $9,5 \pm 6,0 ; \mathrm{p}<0,0001$ et $41 \pm 18,9$ versus $35,25 \pm 17,0 ; \mathrm{p}=0,048$, respectivement).

Pour le patient, la voie radiale est significativement plus irradiante que la voie fémorale pour les 2 types de procédures, coronarographie et coronarographie plus angioplastie ad hoc $\left(59 \pm 50,6 \mathrm{~Gy} \mathrm{~cm}^{2}\right.$ versus $37,5 \pm 24,2 \mathrm{~Gy} \mathrm{~cm}^{2} ; \mathrm{p}<0,0001$ et $123 \pm 77,5 \mathrm{~Gy} \mathrm{~cm}^{2}$ versus $102 \pm 75,7 \mathrm{~Gy} \mathrm{~cm}^{2} ; \mathrm{p}=0,006$, respectivement).

Pour l'opérateur, la voie radiale est également plus irradiante que la voie fémorale en cas de coronarographie et coronarographie plus angioplastie ad hoc $(29 \pm 42,8 \mu \mathrm{Sv}$ versus $13 \pm 28,7 \mu \mathrm{Sv} ; \mathrm{p}=0,0001$ et $69 \pm 97,2 \mu \mathrm{Sv}$ versus $40 \pm$ $76,8 \mu \mathrm{Sv} ; \mathrm{p}=0,043$, respectivement).

Nous trouvons une corrélation statistique :

- par voie radiale :

- entre la dose opérateur et la dose patient : $r=0,68 ; p<0,0001$ et $r=0,61$; $\mathrm{p}<0,0001$ pour les coronarographies et coronarographies plus angioplasties ad hoc respectivement ;

- entre la dose opérateur et le temps de scopie : $\mathrm{r}=0,58 ; \mathrm{p}<0,0001$ pour les coronarographies et $\mathrm{r}=0,46 ; \mathrm{p}<0,0001$ pour les coronarographies et coronarographies plus angioplasties ad hoc respectivement ;

- entre l'irradiation du patient et le temps de procédure : $r=0,70 ; p<0,0001$ pour les coronarographies et $r=0,69 ; p<0,0001$ pour les coronarographie plus angioplasties ad hoc ;

- de façon similaire l'irradiation de l'opérateur est corrélée avec le temps de procédure : $\mathrm{r}=0,71 ; \mathrm{p}<0,0001$ pour les coronarographies et $\mathrm{r}=0,72$; $\mathrm{p}<0,0001$ pour les coronarographie plus angioplasties ad hoc;

- finalement nous trouvons une corrélation entre le BMI (index de masse corporelle) et le PDS (produit dose-surface) du patient pour les coronarographies seules par voie radiale uniquement : $r=0,53 ; p<0,0001$;

- par voie fémorale :

- entre la dose opérateur et la dose patient: par voie fémorale, $r=0,62$; $\mathrm{p}<0,0001$ et $\mathrm{r}=0,50 ; \mathrm{p}<0,0001$ pour coronarographies et coronarographies plus angioplasties ad hoc respectivement;

- entre la dose opérateur et le temps de scopie : $\mathrm{r}=0,47 ; \mathrm{p}<0,0001$ pour les coronarographie plus angioplasties ad hoc;

- entre l'irradiation du patient et le temps de procédure : $r=0,51 ; p<0,0001$ pour les coronarographies et $\mathrm{r}=0,79 ; \mathrm{p}<0,0001$ pour les coronarographie plus angioplasties ad hoc; 
- de façon identique l'irradiation de l'opérateur est corrélée avec le temps de procédure : $\mathrm{r}=0,55 ; \mathrm{p}<0,0001$ pour les coronarographies et $\mathrm{r}=0,67$; $\mathrm{p}<0,0001$ pour les coronarographie plus angioplasties ad hoc.

$\mathrm{Nb}$ : une corrélation existe si $\mathrm{r}>0,4$ et $\mathrm{p}<0,01$. Les résultats de la dosimétrie opérationnelle et passive sous le tablier plombé ont montré des doses très faibles (de 1 à quelques $\mu \mathrm{Sv}$ ).

\section{Interprétation et discussion}

Suivant les publications internationales (Von Boetticher et al., 2003 ; Kiemeneij et al., 1997), il apparaît que la voie radiale est à privilégier sur la voie fémorale car elle engendre beaucoup moins de problèmes hémorragiques. Le bénéfice apporté aux patients subissant une coronarographie et/ou angioplastie coronaire sur ce plan est très important.

Néanmoins les résultats de ce registre dosimétrique comparatif entre la voie radiale droite et la voie fémorale droite, dans la pratique cardiologique interventionnelle, nous montrent une irradiation plus conséquente du patient lors d'examens réalisés par voie radiale droite que par voie fémorale droite ; ceci est valide pour les gestes diagnostiques et également pour les gestes interventionnels. Il existe un facteur d'environ 1,5 entre les deux voies d'abord.

Le PDS $\left(\mathrm{Gy} \mathrm{cm}^{2}\right)$ est en moyenne plus élevé de $36,4 \%$ pour les coronarographies et de $17,1 \%$ pour les coronarographies plus angioplastie ad hoc, cet incrément n'est pas négligeable.

Le comparatif des temps de scopie et des temps de procédure nous montre également des temps plus longs par voie radiale que par voie fémorale.

Pour l'opérateur les doses relevées sur le dosimètre bras sont également plus conséquentes par voie radiale que par voie fémorale. Ces résultats dans leur ensemble confirment les publications internationales (Louvard et al., 2001 ; Lange et Von Boetticher, 2006 ; Sandborg et al., 2004 ; Lotan et al., 1995 ; Hudelo et al., 2000) relatant les difficultés de cathétérisme des coronaires par voie radiale et la différence avec la voie fémorale.

Notons bien que dans notre registre, l'utilisation d'un dosimètre bras pour l'opérateur (inhibition du phénomène de réponse angulaire) n'a été, jusqu'à présent, encore jamais utilisée.

$\grave{A}$ titre de rapprochement, nous avons mis en comparaison les PDS $\left(\mathrm{Gy} \mathrm{cm}^{2}\right)$ et les temps de scopie des NRD (niveau de référence diagnostique) européens et de 


\section{TABLEAU III}

Comparatif produit dose $\times$ surface $(\mathrm{PDS})$ au $75^{\mathrm{e}}$ percentile. Comparison of PDS between Reims Team, GACI data and European reference data at 75 th percentile.

\begin{tabular}{|c|c|c|c|c|}
\hline $75^{\mathrm{e}}$ percentile & Site & Données & $\begin{array}{c}\text { Coronarographie } \\
\text { seule }\end{array}$ & $\begin{array}{c}\text { Coronarographie } \\
+ \text { angioplastie } a d h o c\end{array}$ \\
\hline \multirow{5}{*}{$\begin{array}{l}\text { PDS } \\
\left(\mathrm{Gy} \mathrm{cm}^{2}\right)\end{array}$} & Reims & Radiale & 88 & 191 \\
\hline & & Fémorale & 52,25 & 129,25 \\
\hline & & Global & 73 & 171 \\
\hline & GACI-CAATS* & $\begin{array}{l}\text { Niveau } \\
\text { de l'enquête }\end{array}$ & 56 & 110 \\
\hline & $\mathrm{EU}^{* *}$ & NRD & 57 & 94 \\
\hline
\end{tabular}

\section{TABLEAU IV}

Comparatif des temps de scopie (en min) au $75^{\mathrm{e}}$ percentile. Comparison of scopy duration between Reims Team, GACI data and European reference data at 75 th percentile.

\begin{tabular}{lcccc}
\hline $75^{\mathrm{e}}$ percentile & Site & Données & $\begin{array}{c}\text { Coronarographie } \\
\text { seule }\end{array}$ & $\begin{array}{c}\text { Coronarographie } \\
\text { angioplastie } a d \text { hoc }\end{array}$ \\
\hline $\begin{array}{l}\text { Temps } \\
\text { de scopie } \\
(\mathrm{min})\end{array}$ & Reims & Radiale & 5,9 & 13,4 \\
& & Fémorale & 2,3 & 9,32 \\
& GACI-CAATS* & Global & 4,7 & 11,2 \\
& Niveau & 7 & 15 \\
& EU** l'enquête & NRD & 6 & 16 \\
\hline
\end{tabular}

Source : * Dr O. Bar, C. Maccia, Enquête « GACI-CAATS $2006 » ; * *$ V. Neofotistou (2003) Preliminary reference levels in interventional cardiology, Eur. Radiol. 13, 2259-2263.

NRD : niveau de référence diagnostique.

l'enquête du GACI (Groupe Athérome et Cardiologie Interventionnelle) avec ceux de cette étude (Tabs. III et IV).

Nous constatons que dans notre centre, pour ce qui concerne le PDS par voie fémorale pour les coronarographies et coronarographies plus angioplastie ad hoc nous nous rapprochons des NRD EU et des niveaux de l'enquête du GACI. Par contre nous constatons que nous nous en éloignons avec la pratique radiale ( $c f$. Tab. III). De cette façon, nous pouvons nous interroger sur notre pratique radiale.

Le bénéfice vasculaire périphérique pour le patient d'une voie radiale est il préférable à une irradiation plus conséquente ? 
Nous pouvons constater par ailleurs que les temps de scopie recueillis dans notre centre sont inférieurs aux NRD et niveaux de l'enquête ; ceci prouve la qualité des procédures réalisées par nos opérateurs (rappelons qu'ils possèdent pour chacun d'entre eux une expérience de plus 5 ans par les deux voies d'abord avec au minimum 300 coronarographies et 150 angioplasties annuelles chacun). Ainsi malgré un temps de scopie plus faible, nous retrouvons un PDS plus élevé en radial et équivalent en fémoral. Ceci nous incite à penser que la qualité de notre appareillage radiologique est en cause (amplificateur de luminance âgé), d'ou l'impérative nécessité d'avoir un contrôle du PDS ainsi qu'un suivi qualitatif de la chaîne radiologique, ceci pour abaisser au plus bas les doses reçues par le patient lors de ce type de procédure.

Le temps de procédure est significativement plus important par voie radiale que par voie fémorale. Il faut donc s'orienter vers une voie fémorale si une procédure est présumée difficile. La principale complication clinique chez le patient après une voie d'abord fémorale est la survenue de complications hémorragiques aux points de ponction. L'hémostase artérielle peut être optimisée par l'utilisation de systèmes de fermeture artérielle. Faut-il dans cette optique encourager l'industrie à développer de tels dispositifs de fermetures percutanées qui soient les plus performants possibles afin de permettre l'utilisation de la voie fémorale et ainsi d'optimiser la radioprotection du patient en abaissant au maximum les doses reçues.

En effet, ce type de fermeture permet d'obturer immédiatement après la coronarographie l'orifice de l'artère fémorale de manière percutanée pour limiter la survenue de complications hémorragiques; la voie fémorale devient alors équivalente à la voie radiale sur ce plan. De cette façon, la voie fémorale deviendrait plus attrayante à tout point de vue.

De toute évidence il est opportun de bien cibler l'indication du geste par voie radiale et de ne pas avoir une attitude systématique : il ne faut pas sous prétexte d'éviter la complication hémorragique de privilégier la voie radiale.

En ce qui concerne la radioprotection des opérateurs, nous trouvons donc une corrélation statistiquement significative entre la dose estimée pour le patient et la dose bras opérateur, et confirmons donc que ces deux éléments sont étroitement liés (Tsapaki et al., 2005). Nous constatons ainsi que la dose bras estimée pour l'opérateur est aussi plus conséquente par voie radiale que par voie fémorale. Les 4 opérateurs ayant bénéficié d'une formation spécifique de radioprotection prenaient soin par voie fémorale et voie radiale de respecter la règle de l'éloignement maximal avant chaque scopie ou graphie, en fonction des contraintes techniques liées à l'examen en cours. Néanmoins nous expliquons en 


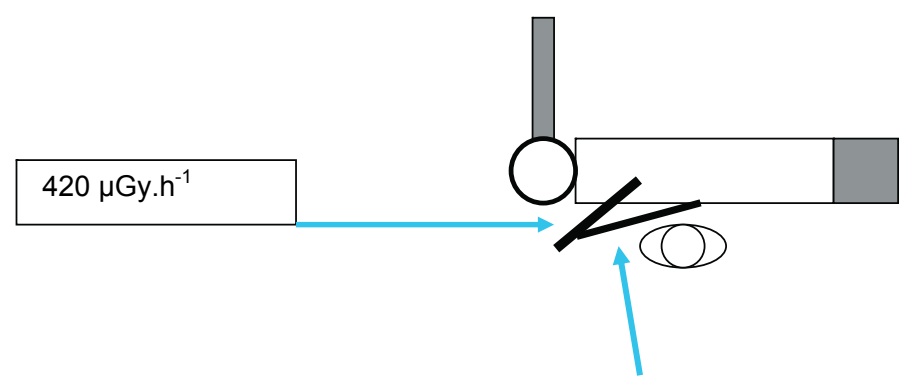

$14 \mu \mathrm{Gy} \cdot \mathrm{h}^{-1}$

Figure 2 - Différence des débits de dose en fonction de la position.

Different acute radiation exposition doses according to position.

partie l'augmentation de la dose opérateur par le fait que les difficultés de cathétérisme engendrés par la voie radiale rapproche plus longuement l'opérateur de l'amplificateur et du patient lors de la recherche des origines des artères coronaires sous scopie : ce qui explique un temps de scopie plus conséquent par voie radiale.

Plus on optimisera la radioprotection du patient, plus on optimisera la radioprotection de l'opérateur. Plus on réduira les temps de procédure et de scopie, plus cela sera bénéfique pour le patient et l'opérateur ; l'opérateur, dans son choix de voie d'abord doit toujours penser à cela.

Une étude de poste de la radio-exposition du personnel a été réalisée sur l'installation radiologique dans notre service, elle montre que le débit de dose devant la vitre plombée est estimé à $420 \mu \mathrm{Gy} \mathrm{h}^{-1}$ alors qu'il est de $14 \mu \mathrm{Gy} \mathrm{h}{ }^{-1}$ derrière la vitre plombée (Fig. 2). Nous avons un rapport de 30 entre les deux débits de dose. Ainsi, nous estimons que l'exposition de l'opérateur serait 30 fois plus importante sans écran radio-atténuateur et bas volet plombé. Avec notre recueil de données, nous pouvons faire une projection de la dose individuelle annuelle estimée au niveau du bras gauche de l'opérateur avec et sans protection. Ceci pour un nombre de 250 et 400 examens, complétés d'un geste interventionnel, annuels et individuels, les résultats sont fournis dans le tableau V.

Selon le tableau V, nous constatons que les doses bras individuelles estimées sans protection pour une activité de 250 procédures annuelles avoisineraient les $500 \mathrm{mSv}$ pour cette voie radiale et $300 \mathrm{mSv}$ pour la voie fémorale et pour 400 procédures annuelles, $828 \mathrm{mSv}$ et $480 \mathrm{mSv}$ respectivement pour la voie radiale et 
TABLEAU V

Comparaison de l'exposition (en $\mathrm{mSv}$ ) de l'opérateur avec et sans protection pour un examen et pour 250 et 400 examens/an selon les 2 approches (fémorale et radiale).

Comparison of operator's exposition between the use and the lack of protective devices for coronary angiogram followed by ad-hoc PCI, according to 250 and 400 procedures each year and the both arterial approaches (femoral and radial).

\begin{tabular}{|c|c|c|c|}
\hline \multirow{2}{*}{\multicolumn{4}{|c|}{$\begin{array}{c}\text { Estimations des doses bras (mSv) avec et sans écran de protection (jupe et tablier plombés) pour } \\
\text { coronarographie }+ \text { angioplastie ad hoc } \\
\text { Rapport des débits de dose derrière et devant protection plombée }=30^{*}\end{array}$}} \\
\hline & & & \\
\hline Dose bras & Avec écran (mSv) & Sans écran (mSv) & \\
\hline $\begin{array}{l}\text { Valeur médiane } \\
\text { fémorale/examen }\end{array}$ & 0,04 & 1,2 & $\begin{array}{l}\mathrm{LAE}(\mathrm{mSv})^{* *} \\
\text { corps entier }\end{array}$ \\
\hline 250 ex/an & 10 & 300 & \multirow{2}{*}{20} \\
\hline 400 ex/an & 16 & 480 & \\
\hline $\begin{array}{l}\text { Valeur médiane } \\
\text { radial/examen }\end{array}$ & 0,069 & 2,07 & $\begin{array}{l}\text { LAE (mSv) } \\
\text { extrémités }\end{array}$ \\
\hline $250 \mathrm{ex} / \mathrm{an}$ & 17,25 & 517,5 & \multirow{2}{*}{500} \\
\hline 400 ex/an & 27,6 & 828 & \\
\hline
\end{tabular}

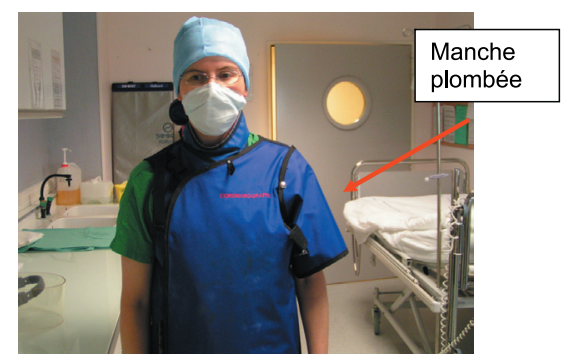

Photo 7.

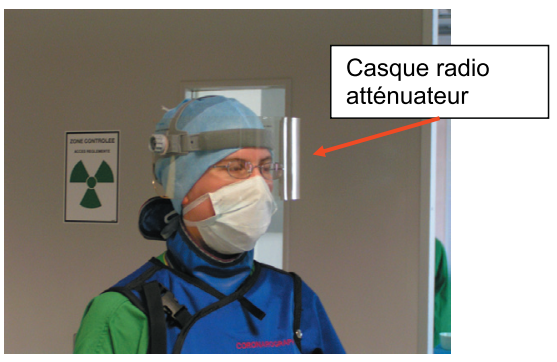

Photo 8.

la voie fémorale: ce qui n'est pas concevable en terme de radioprotection, puisque la LAE pour les extrémités est de $500 \mathrm{mSv}$ et que nous nous trouvons sur le bras proche du creux axillaire.

Ainsi il est licite d'utiliser des tabliers avec manche plombée amovible et interchangeable de façon à protéger le bras ainsi que le creux axillaire de l'opérateur le plus proche de l'amplificateur de brillance (Photo 7). Il parait indispensable d'utiliser bas volet et vitre plombés (Photos 5 et 6).

Nous avons néanmoins validé le fait que le paramètre opérateur n'influait pas sur les résultats en terme d'irradiation pour le patient, mais également pour l'opérateur lui même, pour l'ensemble des procédures, mais aussi pour les coronarographies seules et les coronarographies suivies d'une angioplastie ad hoc, en montrant l'absence de différence significative pour les 4 opérateurs (Tab. VI). 


\section{TABLEAU VI}

Exposition des opérateurs (en $\mu \mathrm{Sv}$ ), des patients (en $\mathrm{Gy} \mathrm{cm}^{2}$ ), durées des procédures (en min) et temps de scopie (en min) en fonction des différents opérateurs pour l'ensemble des procédures et pour chacune des voies d'abord (radiale et fémorale).

Operator's and patient's radiation exposure, duration procedures and scopy durations according to operators for the overall procedures, radial approach procedures and femoral approach procedures.

\begin{tabular}{|c|c|c|c|c|c|}
\hline & Opérateur 1 & Opérateur 2 & Opérateur 3 & Opérateur 4 & $\mathbf{p}$ \\
\hline Toutes procédures $(n=420)$ & $\mathrm{n}=135$ & $\mathrm{n}=133$ & $\mathrm{n}=88$ & $\mathrm{n}=64$ & \\
\hline Irradiation des opérateurs $(\mu \mathrm{Sv})$ & $\begin{array}{c}21,0 \\
{[1,0-445,0]}\end{array}$ & $\begin{array}{c}34,0 \\
{[1,0-531,0]}\end{array}$ & $\begin{array}{c}37,5 \\
{[2,0-265,0]}\end{array}$ & $\begin{array}{c}49,0 \\
{[1,0-283,0]}\end{array}$ & 0,53 \\
\hline Irradiation des patients $\left(\mathrm{Gy} \mathrm{cm}^{2}\right)$ & $\begin{array}{c}64,0 \\
{[17,0-383,0]}\end{array}$ & $\begin{array}{c}62,0 \\
{[20,0-398,0]}\end{array}$ & $\begin{array}{c}89,0 \\
{[10,0-263,0]}\end{array}$ & $\begin{array}{c}75,5 \\
{[10,0-358,0]}\end{array}$ & 0,37 \\
\hline Durées de procédure (min) & $\begin{array}{c}19,0 \\
{[4,0-90,0]}\end{array}$ & $\begin{array}{c}21,0 \\
{[4,0-86,0]}\end{array}$ & $\begin{array}{c}20,3 \\
{[2,0-71,0]}\end{array}$ & $\begin{array}{c}16,0 \\
{[6,0-103,0]}\end{array}$ & 0,55 \\
\hline Temps de scopie (min) & $\begin{array}{c}4,3 \\
{[0,5-30,9]}\end{array}$ & $\begin{array}{c}4,8 \\
{[0,8-26,5]}\end{array}$ & $\begin{array}{c}5,0 \\
{[0,8-28,0]}\end{array}$ & $\begin{array}{c}4,4 \\
{[1,0-34,8]}\end{array}$ & 0,74 \\
\hline Voie radial $(n=239)$ & $\mathrm{n}=53$ & $\mathrm{n}=76$ & $\mathrm{n}=66$ & $\mathrm{n}=44$ & \\
\hline Irradiation des opérateurs $(\mu \mathrm{Sv})$ & $\begin{array}{c}30,0 \\
{[1,0-445,0]}\end{array}$ & $\begin{array}{c}37,5 \\
{[1,0-531,0]}\end{array}$ & $\begin{array}{c}39,5 \\
{[4,0-265,0]}\end{array}$ & $\begin{array}{c}57,5 \\
{[1,0-283,0]}\end{array}$ & 0,81 \\
\hline Irradiation des patients $\left(\mathrm{Gy} \mathrm{cm}^{2}\right)$ & $\begin{array}{c}79,0 \\
{[18,0-334,0]}\end{array}$ & $\begin{array}{c}65,0 \\
{[20,0-398,0]}\end{array}$ & $\begin{array}{c}95,0 \\
{[15,0-263,0]}\end{array}$ & $\begin{array}{c}81,0 \\
{[17,0-358,0]}\end{array}$ & 0,64 \\
\hline Durées de procédure (min) & $\begin{array}{c}25,0 \\
{[4,5-90,0]}\end{array}$ & $\begin{array}{c}22,0 \\
{[4,0-86,0]}\end{array}$ & $\begin{array}{c}21,3 \\
{[5,0-71,0]}\end{array}$ & $\begin{array}{c}20,0 \\
{[6,0-95,0]}\end{array}$ & 0,43 \\
\hline Temps de scopie (min) & $\begin{array}{c}5,9 \\
{[1,2-28,4]}\end{array}$ & $\begin{array}{c}5,5 \\
{[1,1-26,5]}\end{array}$ & $\begin{array}{c}5,5 \\
{[1,0-28,0]}\end{array}$ & $\begin{array}{c}5,2 \\
{[1,1-34,8]}\end{array}$ & 0,93 \\
\hline Voie fémoral $(\mathrm{n}=181)$ & $\mathrm{n}=82$ & $\mathrm{n}=57$ & $\mathrm{n}=22$ & $\mathrm{n}=20$ & \\
\hline Irradiation des opérateurs $(\mu \mathrm{Sv})$ & $\begin{array}{c}17,5 \\
{[1,0-360,0]}\end{array}$ & $\begin{array}{c}30,0 \\
{[2,0-245,0]}\end{array}$ & $\begin{array}{c}20,5 \\
{[2,0-164,0]}\end{array}$ & $\begin{array}{c}16,0 \\
{[1,0-200,0]}\end{array}$ & 0,69 \\
\hline Irradiation des patients $\left(\mathrm{Gy} \mathrm{cm}^{2}\right)$ & $\begin{array}{c}50,0 \\
{[17,0-383,0]}\end{array}$ & $\begin{array}{c}57,0 \\
{[21,0-276,0]}\end{array}$ & $\begin{array}{c}43,0 \\
{[10,0-217,0]}\end{array}$ & $\begin{array}{c}55,0 \\
{[10,0-351,0]}\end{array}$ & 0,93 \\
\hline Durées de procédure (min) & $\begin{array}{c}16,5 \\
{[4,0-90,0]}\end{array}$ & $\begin{array}{c}21,0 \\
{[4,0-64,0]}\end{array}$ & $\begin{array}{c}15,3 \\
{[2,0-54,0]}\end{array}$ & $\begin{array}{c}13,5 \\
{[7,0-103,0]}\end{array}$ & 0,67 \\
\hline Temps de scopie (min) & $\begin{array}{c}3,1 \\
{[0,5-30,9]}\end{array}$ & $\begin{array}{c}3,3 \\
{[0,8-18,4]}\end{array}$ & $\begin{array}{c}3,1 \\
{[0,8-9,4]}\end{array}$ & $\begin{array}{c}2,4 \\
{[1,0-21,4]}\end{array}$ & 0,79 \\
\hline
\end{tabular}

Variables exprimées comme médianes [min-max]. La différence entre les opérateurs est testée en utilisant un test d'ANOVA complété par un test post-hoc de Bonferroni.

D'autre part, l'utilisation de seringue d'injection automatique (type ACIST) permet à l'opérateur, au moment des acquisitions numériques, de s'éloigner de la source et ainsi de diminuer les doses reçues par voie radiale et fémorale.

Par ailleurs, il existe encore trop de salles de cathétérisme cardiaque en France et en Europe qui ne possèdent pas ce type de protections. Il serait envisageable de 
surveiller plus exhaustivement les pratiques dans ces salles, et d'une manière plus générale sensibiliser les cardiologues interventionnels à optimiser leur pratique en terme de radioprotection.

Enfin, nous avons constaté que les doses sous le tablier de l'opérateur (dosimétrie passive et opérationnelle) sont faibles, ceci nous montre l'efficacité des moyens utilisés pour optimiser la radioprotection de l'opérateur.

\section{Conclusions}

Malgré son intérêt en terme d'économie de complication de voie d'abord, la voie radiale droite est significativement plus irradiante pour les patients et les opérateurs que la voie fémorale droite. Son indication doit être repensée dans le contexte de la préoccupation de la radioprotection suivant le principe ALARA ${ }^{3}$.

Nos résultats incitent à souligner l'intérêt d'optimiser cette dernière en utilisant au mieux le matériel disponible : tablier plombé, vitre et bas volet plombés et manche plombée, casque radio atténuateur (Photos 7 et 8 )

D'autre part, nos données nous amènent à confirmer l'intérêt des contrôles de qualité des installations et la nécessité de formation en termes de radioprotection mais également en terme de pratique, des personnels intervenants aux salles de cardiologie interventionnelle.

Un travail de fond doit être mené, alliant la personne spécialisée en radiophysique médicale, la personne compétente en radioprotection et les opérateurs afin d'optimiser les procédures.

\section{RÉFÉRENCES}

Hudelo C., Maillard L., Quilliet L., Desveaux B., Fichaux O., Delplace C., Grimbert M., Arwidson P. (2000) Comparison of coronary angiography by the left radial and right femoral approach with 4 French catheters, Arch. Mal. Cour Vaiss. 93, 813-819.

Kiemeneij F., Laarmann G.J., Odekerken D., Slagboom T., Van der Wieken R. (1997) A randomized comparison of percutaneous transluminal coronary. Angioplasty by the radial, brachial and femoral approaches: the access study, J. Am. Coll. Cardiol. 29, 1269-1275.

Lange H.W., Von Boetticher H. (2006) Randomized comparison of operator radiation exposure during coronary angiography and intervention by radial or femoral approach, Catheter Cardiovasc. Interv. 67, 12-16

Lotan C., Hasin Y., Mosseri M., Rozenman Y., Admon D., Nassar H., Gotsman M.S. (1995) Transradial approach for coronary angiography and angioplasty, Am. J. Cardiol. 76, 164-167.

\footnotetext{
3 ALARA : As Low As Reasonably Achievable, Principe selon lequel l'exposition de l'homme et de l'environnement au rayonnement ionisant doit être aussi faible que raisonnablement possible.
} 
Louvard Y., Lefevre T., Allain A., Morice M. (2001) Coronary angiography through the radial or the femoral approach: the CARAFE study, Catheter Cardiovasc. Interv. 52, 181-187.

Sandborg M., Fransson S.G., Pettersson H. (2004) Evaluation of patient absorbed doses during coronary angiography and intervention by femoral and radial artery access, Eur. Radiol. 14, $653-658$.

Tsapaki V., Kottou S., Vano E., Parviainen T., Padovani R., Dowling A., Molfetas M., Neofotistou V. (2005) Correlation of patient and staff doses in interventional cardiology, Radiat. Prot. Dosim. 117, 26-29.

Von Boetticher H., Meenen C., Lachmund J., Hoffmann W., Engel H.J. (2003) Radiation exposure to personnel in cardiac catheterization laboraties, Z. Med. Phys. 13, 251-256. 